\title{
Determinação de equações de volume para uma área florestal no Município de
}

\section{Marabá - PA}

\author{
Determination of volume equations for a forest area in the Municipality of Marabá - PA \\ Determinación de ecuaciones de volumen para un área forestal en el Municipio de Marabá - PA
}

Recebido: 25/07/2021 | Revisado: 29/07/2021 | Aceito: 14/09/2021 | Publicado: 15/09/2021

Klewton Adriano Oliveira Pinheiro

ORCID: https://orcid.org/0000-0003-2696-4249 Instituto Federal de Educação, Ciência e Tecnologia do Pará, Brasil

E-mail: klewton.pinheiro@gmail.com

Francimary da Silva Carneiro

ORCID: https://orcid.org/0000-0002-1693-8779 Secretaria de Estado de Meio Ambiente e Sustentabilidade, Brasil

E-mail: francimarycarneiro@gmail.com

Marcio Braga Amorim

ORCID: https://orcid.org/0000-0001-7508-2766 Secretaria de Estado de Meio Ambiente e Sustentabilidade, Brasil E-mail: marciobamorim@gmail.com

Carla Vanessa Borges Castro

ORCID: https://orcid.org/0000-0002-5066-0862 Secretaria de Estado de Meio Ambiente e Sustentabilidade, Brasil E-mail: carlavbcastro@yahoo.com.br

Glayson Francisco Bezerra das Chagas

ORCID: https://orcid.org/0000-0002-5796-1924

Secretaria de Estado de Meio Ambiente e Sustentabilidade, Brasil

E-mail: chagasmeteorologista@gmail.com

Bianca Chaves Marcuartú

ORCID: https://orcid.org/0000-0002-5998-760X

Secretaria de Estado de Meio Ambiente e Sustentabilidade, Brasil

E-mail: bianca_marcuartu@hotmail.com

Paula Magno do Amaral

ORCID: https://orcid.org/0000-0002-1749-791X

Secretaria de Estado de Meio Ambiente e Sustentabilidade, Brasil

E-mail: magno_ana@yahoo.com.br

Williams Dias De Oliveira

ORCID: https://orcid.org/0000-0002-2704-3014

Secretaria de Estado de Meio Ambiente e Sustentabilidade, Brasil

E-mail: williamsdoliveira@hotmail.com

Ellen Gabriele Pinto Ribeiro

ORCID: https://orcid.org/0000-0001-9907-3928

Secretaria de Estado de Meio Ambiente e Sustentabilidade, Brasil E-mail: ellengpr@gmail.com

\begin{abstract}
Resumo
As florestas de terra firme, em algumas regiões da Amazônia oriental, assim como em igapós e em várzeas, apresentam alta diversidade de espécies. Estes tipos florestais possuem características diferenciadas que são resultantes das diversas influências dos fatores ambientais. $\mathrm{O}$ trabalho foi realizado na fazenda Pioneira pertencente a Companhia Siderúrgica do Pará entre as latitudes de $5^{\circ} 35^{\prime}$ e $5^{\circ} 55^{\prime}$ (S) e longitude de $48^{\circ} 50^{\prime}$ e $49^{\circ}$ (W), localizado no Município de Marabá no estado do Pará. A mesma disponibiliza de uma área total de 4401,72 ha sendo sua exploração feita em 12 talhões de uma área média de 199,38 ha por talhão. Para atender os objetivos deste trabalho foram selecionadas e cubadas 100 árvores, obedecendo ao critério de balanceamento dentro das classes de ocorrência diametrais. Cada árvore foi seccionada de 2 em 2 metros, medindo-se os diâmetros inicial e final de cada tora de 2 metros e da ponta se fosse o caso. A partir desses dados, utilizando-se o software estatístico SAEG, foram testados vários modelos de regressão, visando obter equações precisas de volumes para a floresta. As equações que melhor se ajustaram aos critérios utilizados foram os modelos de número $6,9,10$ e 11, entretanto a equação 9 , foi a mais indicada por apresentar melhor resultado.
\end{abstract}

Palavras-chave: Floresta de terra firme; Cubagem; Exploração florestal; Região do Carajás. 


\begin{abstract}
The terra firme forests, in some regions of the eastern Amazon, as well as in igapós and floodplains, present a high diversity of species. These forest types have different characteristics that result from the different influences of environmental factors. The work was carried out on the Pioneira farm belonging to Companhia Siderúrgica do Pará between latitudes of $5^{\circ} 35^{\prime}$ and $5^{\circ} 55^{\prime}(\mathrm{S})$ and longitude of $48^{\circ} 50^{\prime}$ and $49^{\circ}(\mathrm{W})$, located in the municipality of Marabá in the state of Pará. It has a total area of 4401.72 ha and its exploration is carried out in 12 plots of an average area of 199.38 ha per plot. To meet the objectives of this work, 100 trees were selected and cubed, following the balancing criterion within the diametrical occurrence classes. Each tree was sectioned every 2 meters, measuring the initial and final diameters of each 2-meter log and the tip, if applicable. From these data, using the SAEG statistical software, several regression models were tested, aiming to obtain precise volume equations for the forest. The equations that best fit the criteria used were models number $6,9,10$ and 11 , however equation 9 , was the most suitable for presenting better results.
\end{abstract}

Keywords: Firm land forest; Cubage; Forest exploration; Carajás region.

\title{
Resumen
}

Los bosques de tierra firme, en algunas regiones de la Amazonía oriental, así como en igapós y llanuras aluviales, presentan una alta diversidad de especies. Estos tipos de bosques tienen diferentes características que resultan de las diferentes influencias de los factores ambientales. El trabajo se llevó a cabo en la finca Pioneira perteneciente a la

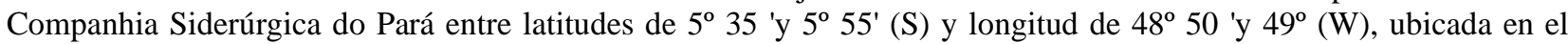
municipio de Marabá en el estado de Pará, tiene una superficie total de 4401,72 ha y su exploración se realiza en 12 parcelas de un área promedio de 199,38 ha por parcela. Para cumplir con los objetivos de este trabajo, se seleccionaron y cubicaron 100 árboles, siguiendo el criterio de balanceo dentro de las clases de ocurrencia diametral. Cada árbol fue seccionado cada 2 metros, midiendo los diámetros inicial y final de cada tronco de 2 metros y la punta, en su caso. A partir de estos datos, utilizando el software estadístico SAEG, se probaron varios modelos de regresión, con el objetivo de obtener ecuaciones de volumen precisas para el bosque. Las ecuaciones que mejor se ajustaron a los criterios utilizados fueron los modelos número $6,9,10$ y 11, sin embargo la ecuación 9 fue la más adecuada para presentar mejores resultados.

Palabras clave: Floresta de tierra firme; Cubagem; Exploração florestal; Região do Carajás.

\section{Introdução}

As florestas de terra firme, em algumas regiões da Amazônia oriental, assim como em igapós e em várzeas, apresentam alta diversidade de espécies. Estes tipos florestais possuem características diferenciadas que são resultantes das diversas influências dos fatores ambientais.

Na Amazônia brasileira, de um modo geral, considera-se o diâmetro de $45 \mathrm{~cm}$ o mínimo para a explorabilidade, motivo pelo qual os volumes individuais das árvores inventariadas são calculados através de equações originadas de dados a partir deste diâmetro de referência, porém, quando se monitora o crescimento volumétrico, especialmente, em florestas manejadas, é conveniente conhecer o crescimento, não só, das árvores grandes, mas também das árvores de tamanho intermediário que constituirão as futuras colheitas (Silva et al .1984).

As espécies dentro desses ecossistemas naturais interagem, entre si, de uma forma bastante dinâmica, resultando em uma estrutura ecológica rica em inter-relações, urgindo a necessidade de estudos sobre a sua composição e sobre a sua estrutura, que são itens importantes para elaboração e aplicação correta de planos de manejo silvicultural, o que permitirá um aproveitamento racional permanente dos recursos florestais, Carvalho(1982), Pinheiro et al (2021), Rodrigues et al(2021), Costa et al(2021), Macedo et al(2021).

De acordo com Barros (1986), as análises devem expressar informações sobre a estrutura dos povoamentos florestais, as quais devem ser baseadas, não somente, em produção volumétrica, mas principalmente, em sua composição e dinâmica florística que constituem os reflexos das diferentes interações dos fatores ambientais. Silva (1984) cita que as florestas secundárias da região Amazônica brasileira constituem uma fonte alternativa de matéria prima para diversas aplicações, tais como madeira serrada, chapas de partículas, celulose e energia dentre outras, e necessitam ter estimativas confiáveis. As informações sobre o volume ou biomassa desse tipo florestal são escassas, especialmente, na Amazônia brasileira, onde a maior ênfase tem sido dada às florestas primárias. Carneiro (2016). 
O principal objetivo do inventário florestal é estimar a quantidade de madeira de uma floresta de acordo com classificações diversas, tais como; espécies ocorrentes, classes de diâmetro e qualidades de fuste (Silva et al, 1984). De acordo com Fernandes (1983), em todas as operações florestais que envolvem a determinação do volume de árvores, principalmente, em inventários florestais, é imprescindível o uso de tabelas de volumes, as quais podem ser elaboradas para determinadas espécies ou grupos de espécies. O volume de madeira de uma floresta quantifica a magnitude que ela representa num todo, embora a determinação dos volumes individuais das árvores requeira precisão, o que é fundamental para determinar o estoque da floresta e calcular a sua produtividade. Baima, (1999), Pinheiro et al (2020).

Sousa et al (1991) cita que a obtenção de uma equação de volume envolve três etapas distintas: amostragem, cubagem e seleção de modelos volumétricos, e para se obter um eficiente ajustamento das equações de regressão, as observações amostrais devem ser colhidas em toda a amplitude de variação dos valores de ocorrência da variável volume.

A análise de resíduo de um modelo de regressão linear possui suma importância na verificação da qualidade dos ajustes de modelos. Os resíduos de um modelo têm uma relação muito forte com a qualidade dos ajustes feito, bem como com a confiabilidade dos testes estatísticos sobre os parâmetros dos modelos (Charnet et al 1999).

Segundo Queiroz (1977), utilizando-se o processo dos mínimos quadrados, através do programa pré-elaborado foi possível testar equações aritméticas e logarítmicas, resultando na seleção da equação logarítmica de Shumacher $\log \mathrm{V}=$ $0,06884+2,02978 \log \mathrm{DAP}+0,75172 \log \mathrm{H}$, por ser a que melhor se ajustou em toda amplitude dos dados de sua pesquisa no planalto de Tapajós.

Queiroz (1984) cita que ao definir o volume sem casca com variável dependente V e o DAP com casca ao quadrado multiplicado pela altura comercial como a variável independente, calculou-se a regressão linear simples V=a+b (DAP2 H), onde os resultados pelo processo dos mínimos quadrados foram: $\mathrm{a}=0,07747596888, \mathrm{~b}=0,517896768, \mathrm{r} 2=0,9652$ e $\mathrm{f}=$ $2994,78$.

Queiroz e Barros (1998) testaram alguns modelos em uma área florestal, no Município de Oriximiná-PA, e encontraram os melhores resultados para a equação $\operatorname{Ln} V=a+b \operatorname{Ln} D A P+c \operatorname{Ln} H$ e $\operatorname{Ln} V=a+b \ln \left(D A P^{2} H\right)$.

A quantificação de uma floresta, através de estudos de volumetria, é uma tarefa importante e deverá ser bem planejada para que a madeira seja mais bem aproveitada, pois, cada vez mais, aumenta no setor florestal a preocupação com os processos de estimação dos volumes de madeira, buscando-se metodologias que aproximem as estimativas, o máximo possível, aos valores reais, e para atingir este objetivo, são desenvolvidas equações de volume por região, que caracteriza melhor a volumetria da área, Pinheiro et al (2020). O presente trabalho tem como objetivo a obtenção de equações de volume para uma floresta situada no Município de Marabá, no Estado do Pará. A maior justificativa para a realização desta pesquisa é que a utilização de equações genéricas para o cálculo dos volumes das árvores, nem sempre são confiáveis, o que determina a necessidade de obter equações apropriadas para cada localidade.

\section{Metodologia}

O trabalho foi realizado na fazenda Pioneira pertencente a Companhia Siderúrgica do Pará entre as latitudes de $5^{\circ} 35^{\prime}$ e $5^{\circ} 55^{\prime}$ (S) e longitude de $48^{\circ} 50^{\prime}$ e $49^{\circ}$ (W), localizado no Município de Marabá no estado do Pará.

A fazenda Pioneira disponibiliza de uma área total de 4401,72 ha sendo sua exploração feita em 12 talhões de uma área média de 199,38 ha por talhão.

Meio Físico

Solos: Segundo publicação dos Estudos Básicos para Planejamento Agrícola - Aptidão Agrícola do Estado do Pará, a área que abrange o plano de manejo florestal localiza-se na mesorregião número 19 caracterizada por latossolo vermelho - 
amarelo distrófico concrecionário, latossolo vermelho - amarelo distrófico e solos concrecionários lateríticos distróficos indiscriminados, sendo que este último não ocorre na propriedade em questão.

Clima: Segundo KOEPPEN, o clima da região é classificado como Am, com temperaturas elevadas durante todo o ano e precipitação em torno de2189 mm anuais, a sua maior concentração está distribuída entre os meses de janeiro a junho. Apresenta, no período seco(verão) deficiência hídrica, principalmente nos meses de setembro a dezembro, época em que os igarapés, na sua maioria secam.

Topografia: O relevo da propriedade bem como da região é ondulado, apresentando-se bastante recortado de igarapés onde tem-se as áreas mais baixas.

Hidrografia: A área do projeto de manejo é bastante recortada por igarapés, caracterizados, em sua grande maioria, por secarem na época de verão (julho a dezembro).

Os principais rios e igarapés que banham a propriedade são: Rio Sororó, Rio Sorozinho, Rio Tauarizinho, Igarapé Patauá, Igarapé Puraqué e Igarapé Água Fria.

\section{Meio Biológico}

Cobertura vegetal: A tipologia vegetal predominante é a floresta densa. Formação arbórea de fisionomia e estrutura variada, caracterizada principalmente, pelo alto porte de seus indivíduos e pela grande variedade de espécies

A floresta densa, conforme a distribuição espacial, reflete nas variações fisionômicas as suas diferenças estruturais.

Para atender os objetivos deste trabalho foram selecionadas e cubadas 100 árvores, obedecendo ao critério de balanceamento dentro das classes de ocorrência diametrais. Cada árvore foi seccionada de 2 em 2 metros, medindo-se os diâmetros inicial e final de cada tora de 2 metros e da ponta se fosse o caso.

De posse destes dados, foi montado um banco de dados planilha e obtidos os volumes reais das árvores, utilizando-se para o cálculo a fórmula de Smaliam $V=[(G f+G g) / 2] \times L$, onde:

$V=$ Volume de tora

$G f=$ Área transversal da parte mais fina da tora.

$G g$ = Área transversal da parte mais grossa da tora.

$L=$ Comprimento da tora

A somatória de todos os volumes obtidos das toras de dois metros, mais o volume da ponta da árvore, resultou no volume real de cada árvore. A partir desses dados, utilizando-se o software estatístico SAEG, foram testados vários modelos de regressão, visando obter equações precisas de volumes para a floresta. Os modelos testados estão relacionados na Tabela 1. 
Tabela 1. Modelos testados para a determinação de equação de volume para a floresta de Marabá.

1) $V=a+b D A P^{2}+c D A P^{2} H^{2}$
2) $V=a+b D A P^{2}+c D A P^{2} H+d H$
3) $V=a+b D A P^{2} H$
4) $V=a+b\left(\frac{1}{D A P}\right)$
5) $V=a+b\left(\frac{1}{D A P}\right)+c D A P$

6) $\operatorname{LnV}=a+b \operatorname{LnDAP}+c \operatorname{Ln} H$

7) $\operatorname{Ln} V=a+b \ln \left(D A P^{2} H\right)$

8) $V=a+b D A P^{2} H+c D A P^{2}+d H$

9) $\log V=a+b \log D A P+c \log H$

10) $\log V=a+b D A P+c D A P^{2}+d D A P^{3}$

11) $\log V=a+b(D A P H)+c(D A P H)^{2}+d(D A P H)^{3}$

12) $V=a+b D A P+c H$

13) $V=a+b D A P^{2}+c D A P^{2} H+d D A P H^{2}+e H^{2}$

14) $V=a+b D A P^{2} H+c H$

Fonte: Autores.

Três critérios foram utilizados para a escolha das melhores equações: alto coeficiente de determinação ( $\left.\mathrm{R}^{2}\right)$, distribuição dos resíduos padronizados e o valor do QMDesvios da Regressão ou o desvio padrão (S). Para poder-se comparar os valores dos desvios das equações logarítmicas com os das não logarítmicas, foram calculados Índices de Furnival para as primeiras.

\section{Resultados e Discussão}

Na Tabela 2 são mostrados os resultados obtidos com o ajuste das equações. 
Tabela 2. Equações de volume de simples e dupla entrada obtidas na análise de regressão para Marabá.

\begin{tabular}{|c|c|c|}
\hline Equações de Volume & \begin{tabular}{l|}
$\mathbf{R}^{2}$ \\
Ajustado \\
\end{tabular} & F CALC \\
\hline $\begin{array}{l}1- \\
v=-0.111626+0.000742640 D A P^{2}+0.00000022197 D A P^{2} H^{2}\end{array}$ & 0.893037 & $509.29^{* *}$ \\
\hline $\begin{array}{l}2- \\
V=-0.253864+0.000379809 D A P^{2}+0.0000139656 A P^{2} H+0.0271517 H\end{array}$ & 0.922735 & $481.68^{* *}$ \\
\hline$v=0.0210868+0.0000303373 D A P^{2} H$ & 0.886452 & $960.24 * *$ \\
\hline$V=1.36351-19.2300\left(\frac{1}{D A P}\right)$ & 0.413991 & $86.98^{* * *}$ \\
\hline$V=-2.54137+19.1077\left(\frac{1}{D A P}\right)+0,0865667 D A P$ & 0.875290 & $428.13 * *$ \\
\hline 6- $L N V=-10.0827+2.02901 L N D A P+0.867363 L N H$ & 0.935333 & $882.30 * *$ \\
\hline $\operatorname{Ln} V=-1.59022+0.0203908 \operatorname{Ln} D A P^{2} H$ & 0.830959 & $604.63^{* * *}$ \\
\hline 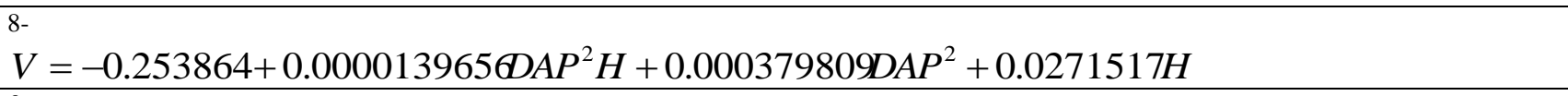 & 0.922735 & $481.68^{* * *}$ \\
\hline ' $\log V=-4.37886+2.02901 \log D A P+0.867363 \log H$ & 0.935333 & $882.30^{* *}$ \\
\hline $\log V=-2.13689+0.0903008 D A P-0.00100697 D A P^{2}+0.00000400430 D A P^{3}$ & 0.922255 & $478.46^{* * *}$ \\
\hline 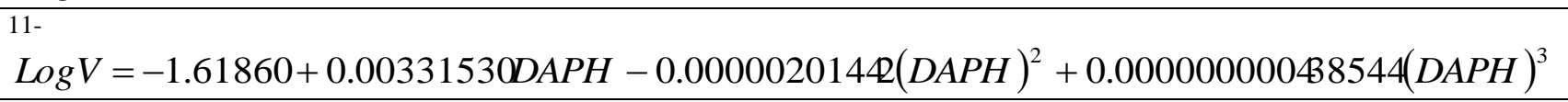 & 0.925352 & $499.98^{* * *}$ \\
\hline$V=-884699+0.0419136 D A P+0.03431126 H$ & 0.825132 & $287.84^{* * *}$ \\
\hline$V=-0.269496-0.00101730 D A P^{2}+0.0000942958 D A P^{2} H-0.000142525 D A P H^{2}+0.00247769 H^{2}$ & 0.909686 & $302.17 * *$ \\
\hline 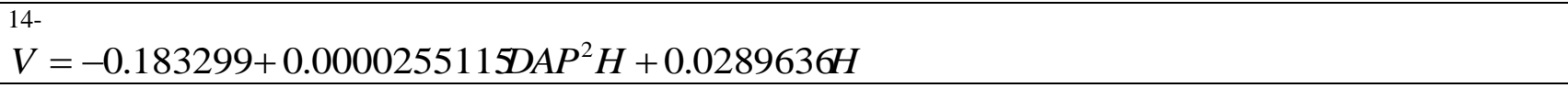 & 0.916093 & $666.00^{* * *}$ \\
\hline
\end{tabular}

Foram observados que as equações de dupla entrada, ou seja, utilizando-se as variáveis DAP e H, preencheram melhor os requisitos de melhor ajustamento em comparação com as de simples entrada, que utiliza apenas o DAP como variável independente. As equações de simples entrada que utilizam apenas o diâmetro das árvores para obtenção do volume, nem sempre oferecem bons resultados, geralmente ocasionando super ou subestimação dos volumes, o que ficou evidente neste trabalho. Conclui-se, mesmo considerando que a variável altura é de difícil medição que as equações de dupla entrada devem ser utilizadas, pois oferecem melhor precisão.

Baima (1999) cita em seu trabalho realizado no Município de Mojú-PA, que a precisão obtida com o ajuste das equações de simples entrada, é inferior quando comparada às equações de dupla entrada. Esta conclusão é devida ser as variáveis diâmetro e altura correlacionados linearmente com a variável volume, principalmente, quando definidas na forma de variável combinada $\mathrm{D}^{2} \mathrm{H}$.

Ainda na tabela 2 verifica-se que as equações logarítmicas 6, 9, 10 e 11 não apresentaram diferenças, em termos de grau de ajustamento, coeficiente de determinação $\left(\mathrm{R}^{2}\right)$, em relação às equações aritméticas 2, 8, 13.e14. 
Tabela 3. Critérios utilizados para julgamento e comparação de equação de volume em ordem de importância.

\begin{tabular}{c|l|l|c}
\hline Equações de volume & QMDesvio da REG. & Desvio Padrão (SI) & Índice de Furnival \\
\hline 1 & 0.03095995 & 0.175954397 & 0.15584149 \\
3 & 0.02428657 & 0.180551627 & \\
4 & 0.03259889 & 0.410169721 & \\
5 & 0.1682392 & 0.189991999 & 0.168331659 \\
6 & 0.03609696 & 0.244260987 & 0.117715418 \\
7 & 0.5966343 & 0.170813289 & 0.073105508 \\
8 & 0.02917718 & 0.150162412 & 0.080488148 \\
10 & 0.02254875 & 0.106081195 & 0.0788688 \\
11 & 0.01125322 & 0.116793921 & 0.114444134 \\
13 & 0.01364082 & 0.224977243 & 0.163023986 \\
\end{tabular}

Fonte: Autores.

A distribuição dos Resíduos em condições ideais é indicada pela diferença entre o valor observado e o valor absoluto para cada árvore, e sua distribuição para indicar qual a mais conveniente deve ser aleatoriamente acima e abaixo dos eixos das abscissas, ou a esquerda e a direita do eixo das ordenadas. A figura 1 apresenta a distribuição dos resíduos dos volumes esperados em função aos volumes observados. Observa-se que os resíduos estão distribuídos, apresentando uma pouca tendência que subestima os valores reais nas classes inferiores e superestima nas classes superiores o que reforça na escolha da melhor equação. 
Figura 1. Distribuição de resíduos do modelo de equação número 9.

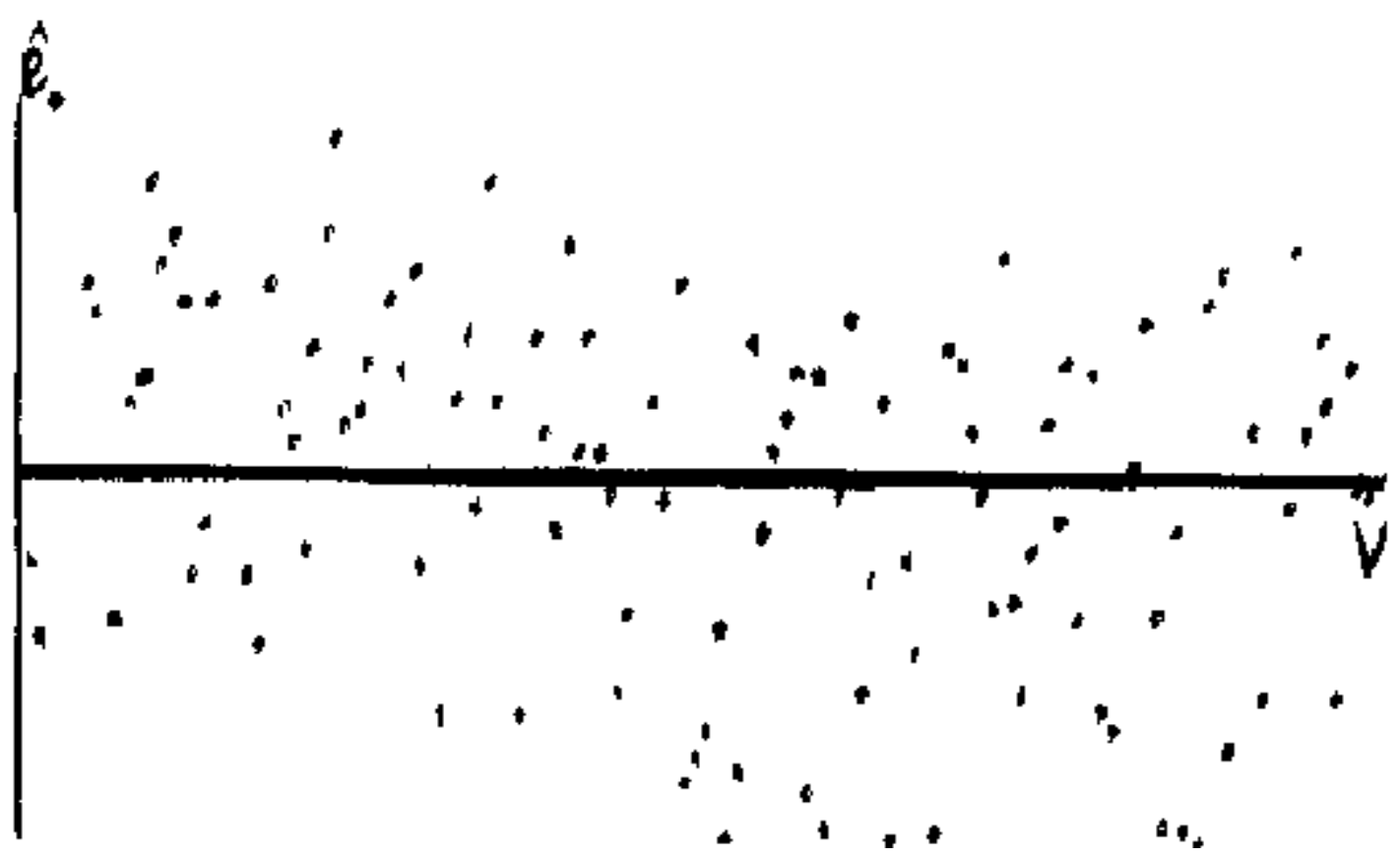

Fonte: Autores.

A distribuição de resíduo representa uma tendência que subestima os valores reais nas classes inferiores e superestima os valores nas classes superiores referente a variável independente

\section{Conclusão}

As equações que melhor se ajustaram aos critérios utilizados foram os modelos de número 6, 9, 10 e 11, entretanto a equação nove foi a mais indicada por apresentar melhores resultados.

\section{Referências}

Baima, A. M. V, Silva, M. A. Da, \& Silva, J. N. M. (1999). Equações de volume para floresta tropical de terra firme do Mojú, PÁ .IN: Simpósio Silvicultural na Amazônia Oriental: Contribuição do projeto Embrapa/DFID. Posters. Embrapa/DFID.

Barros, P.L.C. (1986). Estudos fitossociológicos de uma floresta tropical no planalto de Curuá- Una, Amazônia Brasileira. Curitiba, 158p. Tese (Doutorado). Universidade Federal do Paraná.

Carneiro, F. S., Ruschel, A. R., Oliveira, F. A., Soares, M. H. M., Rodrigues, C. F. A., \& Pinto, M. V. P. (2020). Resiliência da Biomassa Acima do Solo em Áreas Experimentais na Amazônia Oriental. Biodiversidade Brasileira, 74-83.

Carneiro, F. S. (2016). Resiliência florestal pós-colheita na Amazônia oriental brasileira. Belém, 2016.141 f. Tese (Doutorado em Ciências Agrárias) Universidade Federal Rural da Amazônia, Empresa Brasileira de Pesquisa Agropecuária - EMBRAPA, https://ainfo.cnptia.embrapa.b r/digital/bitstream/item/175660/1/TeseFinal-FrancimaryCarneiro-08112017.pdf

Carvalho, J. O. P. (1982). Análise estrutural da regeneração natural em floresta tropical densa na região de Tapajós no Estado de Pará. Curitiba, 129p. Dissertação (Mestrado), Universidade Federal do Paraná.

Charnet, R. et al. (1999). Análise de estudos de regressão linear com aplicações. Editora da Unicamp. (Coleção Livro. Texto).

Costa, S. C. C., Jardim, F. C. S., Carneiro, F. S., Amorim, M. B., \& Pinheiro, K. A. O. (2021). Regeneração de Protium pallidum Cuatrec. em clareiras originadas da exploração madeireira seletiva na Amazônia Oriental Brasileira. Research, Society And Development, 10 , e41510313534.

Fernandes, N. P. et al. (1983). Tabelas de volumes para a floresta de terra firme da Estação Experimental de Silvicultura Tropical. Acta Amazônia, n. 13 (3-4), $537-545$.

Pinheiro, K. A. O., Oliveira, F. A., Ruschel, A. R., Pita, J. D., Santos, G. C., Silva, F. L., Frazão, A. S., Carneiro, F. S., \& Souza, M. F. S. (2020). Volumetric space distribution of wood as a tool in sustainable forest. African Journal of Agricultural Research, 15, 312-322. 
Research, Society and Development, v. 10, n. 12, e131101218690, 2021

(CC BY 4.0) | ISSN 2525-3409 | DOI: http://dx.doi.org/10.33448/rsd-v10i12.18690

Gomes, J. M., Carvalho, J. O. P., Ruschel, A. R., Silva, J. N. M., Soares R. E. M. L., Castro, T. C., Costa, N. S. L., Carneiro, F. S., \& D’Arace, L. M. B. (2021). Regeneração Natural de Espécies Ameaçadas de Extinção em Áreas Experimentais na Amazônia Oriental. Biodiversidade Brasileira, 11, 1-11.

Pinheiro, K. A. O., Carvalho, J. O. P., Schwartz, G., Francez, L. M. B., Quanz, B., \& Carneiro, F. S. (2020). Estrutura de uma comunidade arbórea adulta na Amazônia e o desafio de uso sustentável de espécies florestais comerciais. Revista Ibero-americana de Ciências Ambientais, 11, 63-73.

Pinheiro, K. A. O., Oliveira, F. A., Ruschel, A. R., Carneiro, F. S., Frazão, A. S., Souza, M. F. S., D’Arace, L. M. B., \& Amorim, M. B. (2021). Potencial de espécies comerciais analisado pelo índice de valor de importância em área de exploração de impacto reduzido. Research, Society And Development, 10, e16610212292.

Queiroz, W. T. (1984). Análise de Fatores (Factor Analysis) pelo Método da Máxima Verossimilhaça: Aplicação ao Estudo da Estrutura de Floresta Tropical. Piracicaba: Escola superior de agricultura Luiz de Queiroz. 107p.

Queiroz, W. T. (1977). Efeito de Variação Estrutural em Anuidades Amostrais na explicação do processo de amostragem em conglomerado nas Florestas do Planalto do Tapajós. Curitiba: Universidade Federal do Paraná. 109p.

Queiroz, W. T. \& Barros, A. V. (1998). Inventário Florestal de 3097 ha da Floresta Nacional de Saracá-Tacuera - Município de Oriximiná -Pará. Belém: Mineração Rio do Norte: Faculdade de Ciências Agrárias do Pará. Departamento de Ciências Florestais.173 p.

Rodrigues, C. F. A., Ruschel, A. R., Mendes, F. S., Carneiro, F.S., Santos, J. C., \& Sousa, M. A. R. (2021). Fitossociologia e análise temporal do fragmento florestal urbano Capoeira do Black, Belém, Pará. Research, Society And Development, 10, e11010212301.

Silva, J. N. M. et al. (1984). Equação de volume para a Floresta Nacional do Tapajós. Boletim de Pesquisa Florestal, (8/9), 50-63. https://ainfo.cnptia.embrapa.br/digital/bitstream/CNPF-2009-09/5024/1/jnsilva3.pdf.

Silva, J. N. M. et al. (1984). Equação de volume para árvores de pequeno diâmetro, na Floresta Nacional do Tapajós. Belterra. PÁ. Boletim de Pesquisa Florestal, 8/9, 16-25. https://ainfo.cnptia.embrapa.br/digital/bitstream/CNPF-2009-09/5022/1/jnsilva2.pdf

Silva, J. N. M. et al. (1984). Equação de volume para uma Floresta Secundária no Planalto do Tapajós. Belterra. PÁ. Boletim de Pesquisa Florestal, 8/9, 1- 15,

Sousa, A.L de. et al. (1991). Equação de volume Comercial e Fator de Forma para espécies da Mata Atlântica Ocorrentes na Reserva Florestal da Companhia Vale do Rio Doce, Linhares, ES. Revista Árvore, 15 (3), 257-273. 